\title{
Protective Effect of Cerium Oxide Nanoparticles on Oxaliplatin induced Neurotoxicity in Adult Male Albino Rats
}

\author{
Amer MA ${ }^{1}$, Farahat $\mathrm{FY}^{1}$, Abd El-Khalik $\mathrm{AM}^{1}$ and Hassan $\mathrm{HE}^{2}$ \\ ${ }^{1}$ Department of Forensic Medicine \& Clinical Toxicology, Faculty of Medicine, Zagazig University, \\ Egypt. \\ ${ }^{2}$ Department of Pathology, Faculty of Medicine, Zagazig University, Egypt.
}

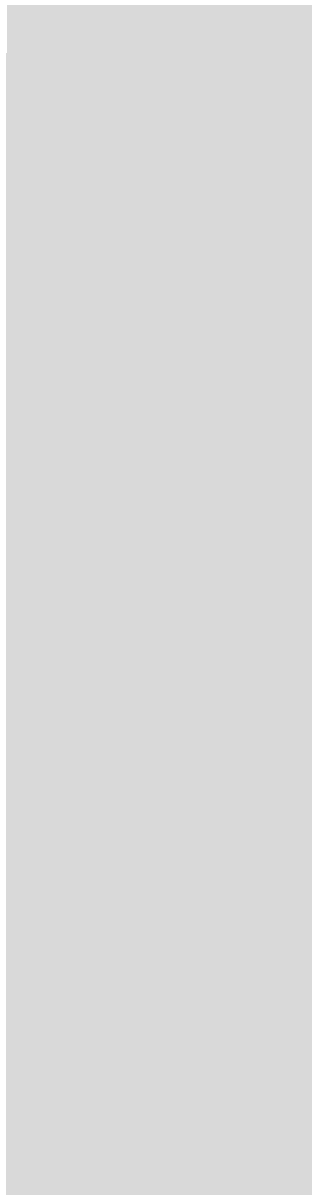

\section{INTRODUCTION}

$\mathbf{O}$ xaliplatin is a third generation platinum based anticancer agent. It is the first line therapy used for the treatment of colorectal cancer (Arnold et al., 2017). The most common side effect of oxaliplatin is peripheral neuropathy which occurs in both acute and chronic forms (Argyriou, 2015). Acute neuropathy occurs within hours after injection in almost all patients and can be resolved within days. Chronic neuropathy occurs in about $75 \%$ of patients after multiple injections of oxaliplatin, which is difficult to be resolved. neuroprotective effect. stress, Myelin protein zero.

\section{ABSTRACT}

Oxaliplatin is a chemotherapeutic agent used for treatment of colorectal cancer. Peripheral neuropathy is a dose limiting toxicity which may persist even after its termination. Oxidative stress may be a direct cause of this neurotoxicity. Cerium oxide nanoparticles (CONPs) are promising antioxidants for applications in medicine. The present work was designed to study the protective effect of CONPs on neurotoxicity induced by oxaliplatin in adult male albino rats. One hundred and twenty male adult albino rats were divided into 4 groups: Control group, CONPs group $(60 \mathrm{mg} / \mathrm{kg}, 5$ times/week), Oxaliplatin group $(4 \mathrm{mg} / \mathrm{kg}$, twice/week) and Oxaliplatin\&CONPs group, for 8 weeks. After $4 \& 8$ weeks, 10 rats from each group were used to measure myelin protein zero (MPZ), malondialdhyde (MDA), superoxide dismutase (SOD), glutathione peroxidase (GPx) and reduced glutathione (GSH) in the left sciatic nerves. The right sciatic nerves and lumbar spinal cord were used for histopathological study. Oxaliplatin decreased MPZ and induced demyelination and degeneration of sciatic nerve fibers. Also, it increased MDA levels. SOD and GPx activity were increased then decreased. GSH levels were decreased. In the lumbar spinal cord sections, abnormal morphology was seen. CONPs administration with oxaliplatin increased MPZ and returned normal rphology of sciatic nerve fibers. They decreased MDA levels. SOD was

Key words: Oxaliplatin, Cerium oxide nanoparticles, Neurotoxicity, Oxidative

This form may persist for months or even years after termination of chemotherapy (Balayssac et al., 2015).

Oxaliplatin induced peripheral neuropathy (OIPN) remains a problematic adverse drug reaction in digestive oncology. To this day, no preventive or curative strategy has proven to be effective because the proper mechanism is still unclear (Cheng et al., 2017).

Oxidative stress may be a direct cause of this neurotoxicity as antineoplastic agents induce apoptosis in tumor cells through generation of reactive oxygen species (ROS). 
These species affect also normal cells and may lead to neurotoxicity (Hershman et al., 2014).

The damage of the peripheral nerves may occur through affection of myelin sheath, dysfunction of mitochondria, inflammation or apoptosis. Also, direct damage of spinal cord by oxaliplatin is suggested to play a role in the mechanism of neuronal affection (Fardell et al., 2015).

Cerium oxide nanoparticles (CONPs) act as direct antioxidants and behave as free radical scavengers. They have properties like that of superoxide dismutase, catalase and peroxidase enzymes (Singh, 2016). They are found to be effective against pathologies associated with chronic oxidative stress and inflammation. CONPs well suited for applications in medicine because they are well tolerated in vitro and in vivo biological models (Das et al., 2013). So, the aim of this work was to study the protective effect of CONPs on neurotoxicity induced by oxaliplatin in adult male albino rats through biochemical and histopatholgoical study of sciatic nerves and lumbar part of spinal cord.

2. Material and Methods

2.1. Material:

Oxaliplatin was purchased as pharmaceutical preparation (100mg/vial), manufactured by Actavis Italy S.P.A. company, Milano, Italy. It was in the form of white powder. Cerium oxide nanopowder was purchased from Sigma/Aldrich chemical company, USA in the form of white nanopowder, <25nm particle size and purity $99.95 \%$. Rat MPZ enzyme-linked immunosorbant assay (ELISA) kit (Bioneovan company, Beijing, China), from Biovision Egypt chemical company. MDA, SOD enzyme, GPx enzyme and GSH kits were purchased from Biodiagnostic chemical company in Egypt.

2.2. Characterization of CONPs by Transmission electron microscope:

Transmission electron microscope (TEM) analysis was carried out for the assessment of primary particle size and morphology of CONPs (JEOL JEM-1400) JEOL Ltd., Tokyo, Japan, operating at an acceleration voltage $80 \mathrm{kV}$. This characterization was done in Electron Microscopy Unite, Faculty of agriculture research park, Cairo university (FARP). The sample of CONPs was prepared by suspending NPs in distilled water. Then the sample was sonicated. A drop of suspension was made on 400 mesh Copper grid coated with a thin layer of carbon and allowed to air dry prior to measurement.

2.3. Animals and grouping:

One hundred and twenty male adult albino rats weighing 180- $200 \mathrm{gm}$, were obtained from the animal house of Faculty of Medicine, Zagazig University. First, all animals subjected to 10 days of passive preliminaries for house acclimatization. Animals were allowed free access to solid food and water in their home cages with proper ventilation. The room was maintained with 12h-light/dark cycle. All experimental procedures were ethically approved by The Ethical committee for scientific research of faculty of Medicine, Zagazig university, in accordance with the guidance of ethical committee for research on laboratory animals (Institute of Laboratory Animal Resources, 1996).

After housing acclimatization, the rats were divided into 4 groups as following :

- Control group (I): Group I was divided into three subgroups (IA, IB, IC), each of 20 rats.

- Negative control group (I A): Rats were kept only on regular diet and tap water to measure the basic parameters.

- Positive control group (IB): Each rat was injected intraperitoneally (IP) with $0.5 \mathrm{ml}$ of $0.9 \%$ sodium chloride solution (vehicle of CONPs) five times per week.

- Positive control group (I C): Each rat was injected IP with $0.5 \mathrm{ml}$ of $5 \%$ glucose solution (vehicle of oxaliplatin) twice per week.

- CONPs group (II) (20 rats): CONPs were suspended in $0.9 \%$ sodium chloride solution and were mixed by sonication, then they were IP injected at a dose of $(60 \mathrm{mg} / \mathrm{Kg}$ body weight $)$ five times per week. The dosage of CONPs was chosen according to (Nigieh et al., 2012).

- Oxaliplatin group (III) (20 rats): Oxaliplatin was dissolved in a $5 \%$ glucose solution at a 
concentration of $2 \mathrm{mg} / \mathrm{ml}$. It was IP injected at a dose of $(4 \mathrm{mg} / \mathrm{Kg}$ body weight) twice per week. LD50 of IP injection of oxaliplatin $=14.3 \mathrm{mg} / \mathrm{kg}$ body weight (Kono et al., 2015).

- Oxaliplatin\&CONPs group (IV) (20 rats): In addition to oxaliplatin injection $(4 \mathrm{mg} / \mathrm{kg}$, IP, twice/week), CONPs were injected IP (60 $\mathrm{mg} / \mathrm{kg}$ ) five times/week. CONPs were injected first, then after 3 hours oxaliplatin was injected (Hirst et al., 2011; Dowding et al., 2014).

The period of the study was 8 weeks. After $4 \& 8$ weeks of the study, 10 rats from each group were anesthetized with sodium pentobarbital $(50 \mathrm{mg} / \mathrm{kg}$ body weight $)$ by IP injection. The sciatic nerves were dissected (Mid- thigh incision approach) (Bala et al., 2014) (figure 1). The left sciatic nerve was homogenized in cold phosphate buffer saline (PBS) solution with $\mathrm{pH} 7.4,5-10 \mathrm{ml}$ per gram tissue, using tissue homogenizer. After homogenization, the homogenate was centrifuged at 4000 r.p.m for $15 \mathrm{~min}$ at $4{ }^{\circ} \mathrm{C}$. The supernatant was collected and stored at $-80{ }^{\circ} \mathrm{C}$, pending biochemical analysis. The right sciatic nerve from each rat was excised and fixed in 10\% neutral formalin for histopathological examination.

The rat spine was harvested en bloc from the T11 vertebra to the L1 vertebra where the lumbar spinal cord located (last rib was the land mark $=\mathrm{T} 13, \mathrm{~T} 13-\mathrm{L} 1$ vertebrae $=\mathrm{L} 4-\mathrm{L} 5$ spinal segments), then it was cleared of the paraspinal muscles and fixed in $10 \%$ neutral formalin then lateral laminectomy was done to each vertebra to clear lumbar spinal cord which post fixed in $10 \%$ neutral formalin for histopathological examination (Wen et al., 2015) (figure 2).

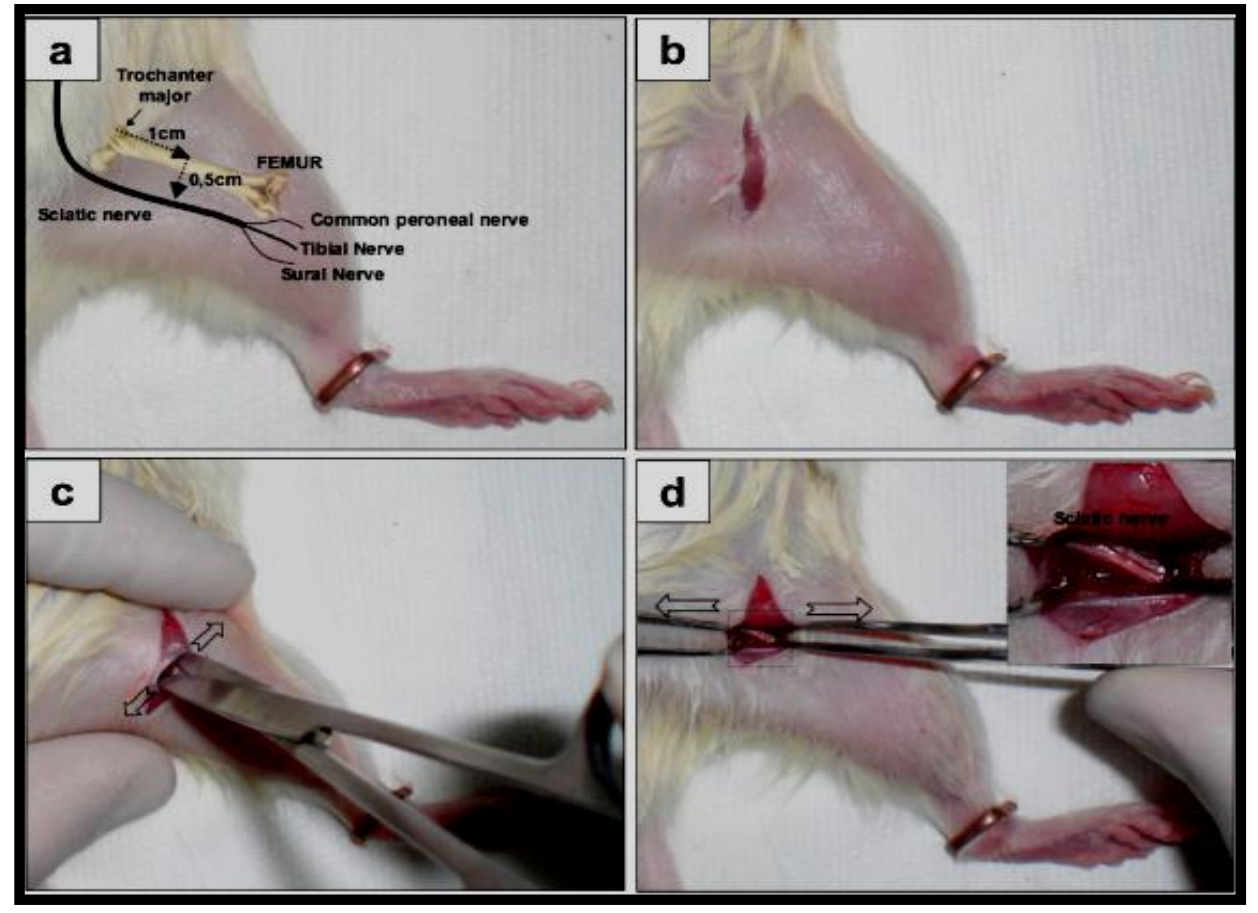

Figure (1): Rhombic dissection between the maximum gluteus and quadriceps muscles was carried out allowing the complete vision of sciatic nerve (Savastano et al., 2014). 


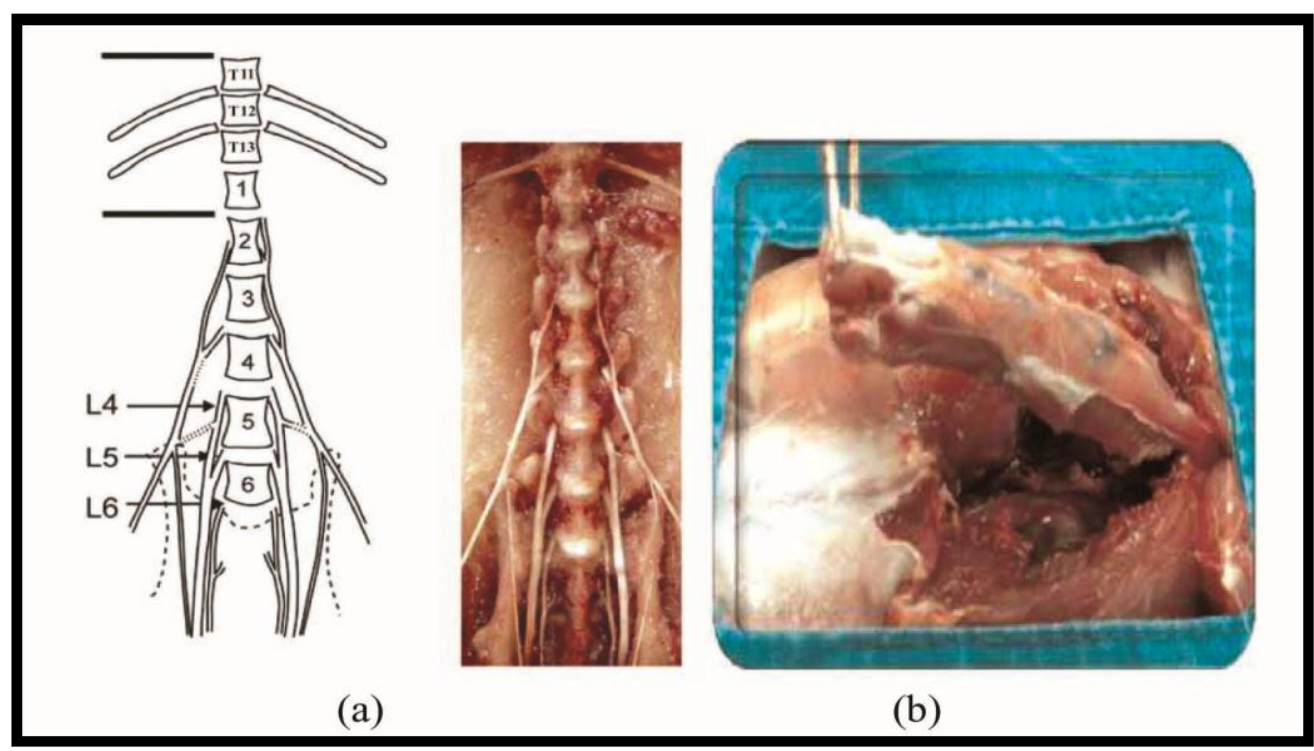

Figure (2): Dissection of the lumbar part of spinal cord. (a) The rat spine was dissected from the T11 vertebra to the L1 vertebra where the lumbar spinal cord located. (b) The rat spine was harvested en bloc (Rigaud et al., 2008).

\subsection{Biochemical Studies:}

The left sciatic nerve homogenate from each rat was subjected to the following:-

2.4.1 Myelin protein zero was assayed by SandwichELISA as the method of (Schmidt et al., 2012).

2.4.2 Oxidative stress markers: MDA, SOD, Gpx enzymes and GSH were assayed colorimetrically as the method of (Ohkawa et al., 1979; Nishikimi et al., 1972; Paglia and Valentine, 1967; Beutler et al., 1963) respectively.

\subsection{Histopathological methods:}

The right sciatic nerve and the lumbar part of the spinal cord of rats from all groups were excised and fixed in $10 \%$ neutral formalin, then dehydrated in $70 \%$ ethanol. Each nerve or lumbar spinal cord was then embedded in3. paraffin. Five $\mu \mathrm{m}$ sections were prepared from the paraffin block and stained by hematoxylin and eosin then were evaluated under light microscope. The processing technique was adapted from Horobin and Bancroft, (1998).
2.6. Statistical Analysis:

Data were collected and analyzed using Statistical Package for Social Sciences (SPSS version 20.0). Quantitative data were done by One Way Analysis of Variance (ANOVA), followed by Post Hoc analysis (Least Significance Difference test "LSD") for multiple comparisons between groups. Qualitative data were done by Chi square test $\left(\mathrm{X}^{2}\right)$. Propability ( $\mathrm{P}$ value) was set as $\mathrm{P}$ value of $>0.05$ indicates non-significant results, $<0.05$ for significant results, $<0.001$ for high significant result and $<0.001$ for very high significant results.

\section{RESULTS}

Characterization of CONPs:-

Transmission electron microscope analysis of CONPs showed mixture of cubes and octahedrons, in the range $<25 \mathrm{~nm}$ with some agglomeration (Figure 3 ). 


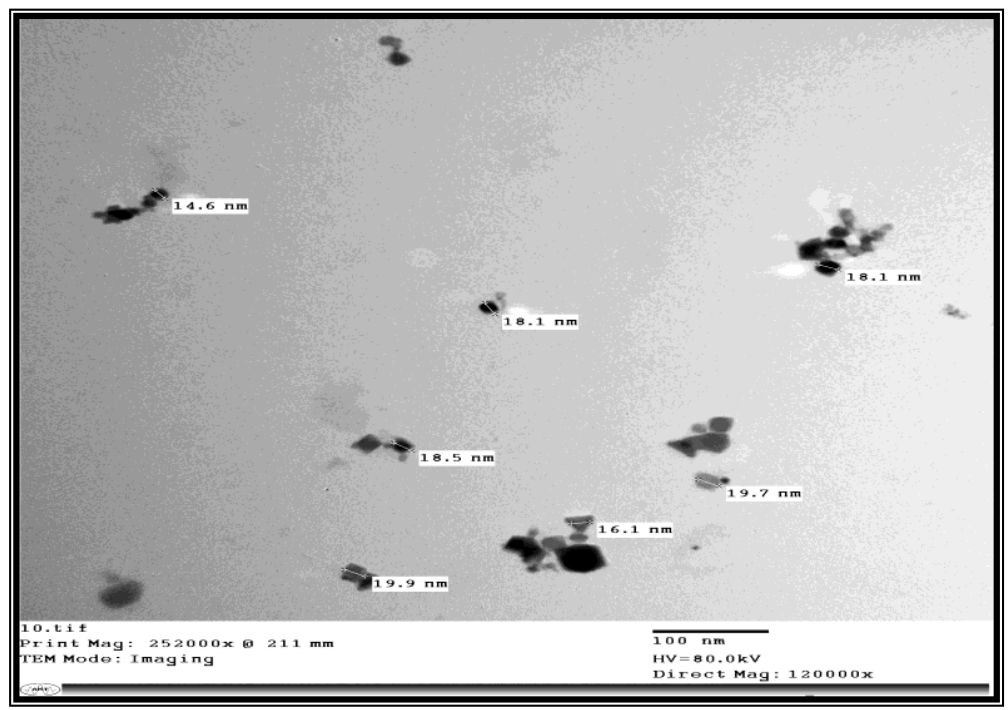

Figure (3): Transmission electron microscope image of nanoparticles of cerium oxide. TEM analysis revealed that CONPs had different shape and size $<25 \mathrm{~nm}$ with some agglomeration.

\subsection{Biochemical results:}

3.2.1 The biochemical parameters of control group was within normal values as regard MPZ, MDA, SOD, GPx \& GSH. There was no statistical significant difference observed so, the negative control group (IA) was used as a control group for comparison with other treated groups.

\subsubsection{Biochemical parameters of treated groups:}

- Myelin protein zero levels:

CONPs alone did not significantly change the MPZ levels when compared to group(IA) $\quad(\mathrm{p}>0.05) . \quad$ Oxaliplatin administration caused a significant decrease in the MPZ levels of sciatic nerve tissues when compared with group(IA) and CONPs group $(\mathrm{p}<0.001)$. The oxaliplatin \& CONPs treated group showed significant increase in levels of MPZ in the sciatic nerve tissues when compared with the oxaliplatin treated group $(p<0.01)$. On the other hand there was no significant difference between oxaliplatin \& CONPs treated group and group (IA) ( $>>0.05)$ (table 1).

- Oxidative stress markers of sciatic nerve tissues:-

\section{Malondialdehyde:}

Oxaliplatin administration caused a high significant increase in the MDA levels of sciatic nerve tissues when compared with group(IA) and CONPs group $(\mathrm{p}<0.001)$. The oxaliplatin \& CONPs treated group showed significantly lower levels of MDA in the sciatic nerve tissues when compared with the oxaliplatin treated group at 4 and 8 weeks of the study $(\mathrm{p}<0.001, \mathrm{p}<0.05)$ respectively. There was no significant difference between oxaliplatin \& CONPs treated group and group (IA) (p> 0.05) (table 2).

Superoxide dismutase enzyme activity:

Oxaliplatin administration for 4 weeks caused a significant increase in the SOD enzyme activity level of sciatic nerve tissues when compared with group (IA) $(\mathrm{p}<0.001)$ and CONPs group $(\mathrm{p}<0.01)$. The oxaliplatin \& CONPs treated group showed non significant lower levels of SOD enzyme when compared to oxaliplatin treated group $(\mathrm{P}>0.05)$ which not returned to normal levels when compared with group (IA) $(\mathrm{p}<0.05)$. On the other hand oxaliplatin administration for 8 weeks caused a highly significant decrease in the SOD enzyme activity levels of sciatic nerve tissues when compared with group (IA) and CONPs group $(\mathrm{p}<0.001)$. The Oxaliplatin \& CONPs treated group showed significantly high levels of SOD enzyme in the sciatic nerve tissues when compared with the oxaliplatin treated group $(\mathrm{p}<$ 
0.001). There was no significant difference between oxaliplatin \& CONPs treated group and group (IA) ( $>>0.05)$ (table 2).

Glutathione peroxidase enzyme activity:

The mean values of GPx enzyme activity level of sciatic nerve tissues of group (IA), CONPs, Oxaliplatin and Oxaliplatin \& CONPs treated group revealed non significant difference between them at 4 weeks of the study $(p>0.05)$. On the other hand oxaliplatin administration for 8 weeks caused significant decrease in the GPx enzyme activity levels of sciatic nerve tissues when compared with the group(IA) \& CONPs group $(\mathrm{p}<0.01)$. Oxaliplatin \& CONPs treated group showed also significantly low levels of GPx enzyme in the sciatic nerve tissues when compared with the $\operatorname{group}(\mathrm{IA}) \quad(\mathrm{p}<0.05)$. There was no significant difference between the oxaliplatin \& CONPs group and oxaliplatin treated group (p>0.05) (table 2).

Reduced glutathione levels:

Oxaliplatin administration for 4 \& 8 weeks caused significant decrease in GSH levels of sciatic nerve tissues when compared with the group (IA) $(\mathrm{p}<0.001)$. The oxaliplatin $\&$ CONPs treated group (IV) showed also low levels of GSH levels in the sciatic nerve tissues when compared with group (IA) $(\mathrm{p}<0.001$, $\mathrm{p}<0.01)$. On the other hand there was no significant difference between the oxaliplatin \& CONPs group and the oxaliplatin treated group $(\mathrm{p}>0.05)$ (table 2).

Table (1): Statistical comparison between negative control group and different treated groups (CONPs cerium oxide nanoparticles, Oxaliplatin and Oxaliplatin \& CONPs) as regard levels of MPZ (Myelin protein zero) at 4 and 8 weeks of the study.

\section{Biochemical \\ parameter}

MPZ

Pg/ml

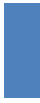

\begin{abstract}
4 weeks
\end{abstract}

\begin{tabular}{|c|c|}
\hline \multirow{2}{*}{8 weeks } & $\begin{array}{c}\text { Conaliplatin (III) } \\
\text { Oxaliplatin\& CONPs(IV) }\end{array}$ \\
\cline { 1 - 2 } & -ve control (IA) \\
CONPs (II) \\
Oxaliplatin (III) \\
\hline Oxaliplatin\& CONPs (IV)
\end{tabular}

Group

$\mathrm{n}=10$

-ve control (IA)

CONPs (II)

CONPs (II)

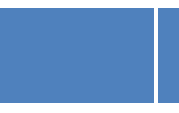

(1)

\begin{tabular}{|c|}
\hline $1723.6 \pm 484.8$ \\
\hline $1711 \pm 232.7$ \\
\hline $1024 \pm 101.9 * * *$ \\
\hline $1523.9 \pm 743.9 \# \#$ \\
\hline $1739 \pm 473.2$ \\
$1785 \pm 321.4$ \\
\hline $923.4 \pm 184.8 * * *$ \\
\hline $1561.6 \pm 748 \# \#$
\end{tabular}

N.B: Values are expressed as Mean \pm SD. (SD: standerd deviation).

n: number (number of sacrificed rats in each group=10 rats).

*** very high significant $(\mathbf{P}<0.001)$ vs. control group; \#\#high significant $(\mathrm{P}<0.01)$ vs. oxaliplatin group. 
Table (2): Statistical comparison between negative control group and different treated groups (CONPs cerium oxide nanoparticles, Oxaliplatin and Oxaliplatin \& CONPs) as regard levels of Malondialdehyde (MDA), Superoxide dismutase (SOD), glutathione peroxidase (GPx) \& reduced glutathione (GSH) at 4 and 8 weeks of the study.

\begin{tabular}{|l|l|l|l|l|}
\hline & -ve control (IA) & CONPs (II) & Oxaliplatin (III) & $\begin{array}{l}\text { Oxaliplatin \& } \\
\text { CONPs (IV) }\end{array}$ \\
\hline $\begin{array}{l}\text { MDA } \\
\text { 4 weeks }\end{array}$ & $118.2 \pm 13.7$ & $119.9 \pm 12.8$ & $188.4 \pm 50.2 * * *$ & $134.8 \pm 27.4 \# \# \#$ \\
\hline 8 weeks & $117.3 \pm 12.4$ & $121.7 \pm 14.1$ & $182.6 \pm 74.4 * * *$ & $146.6 \pm 30.8 \#$ \\
\hline $\begin{array}{l}\text { SOD } \\
\text { 4 weeks }\end{array}$ & $5218.5 \pm 1167.7$ & $5785.7 \pm 905.4$ & $7544.3 \pm 1524.9 * * *$ & $6491.6 \pm 1793.9 *$ \\
\hline $\begin{array}{l}\text { 8 weeks } \\
\text { GPx }\end{array}$ & $9422.3 \pm 33.2$ & $8892.9 \pm 1366.2$ & $6968.8 \pm 1793.9 * * *$ & $8868.5 \pm 529.9 \# \# \#$ \\
\hline $\begin{array}{l}\text { 8 weeks } \text { weeks } \\
\text { GSH } \\
\text { 4 weeks }\end{array}$ & $129.7 \pm 26.7$ & $116.7 \pm 38.9$ & $151.4 \pm 51.8$ & $90.8 \pm 45.7$ \\
\hline $\mathbf{8}$ weeks & $127.7 \pm 36.9$ & $121.3 \pm 38.8$ & $38.5 \pm 12.6 * *$ & $53.2 \pm 22.7 *$ \\
\hline
\end{tabular}

N.B: Values are expressed as Mean \pm SD. (SD: standerd deviation).

n: number (number of sacrificed rats in each group $=10$ rats).

*significan $(\mathbf{p}<0.05), * *$ high significant $(\mathrm{P}<0.01), * * *$ very high significant $(\mathrm{P}<0.001)$ vs. control group; \# significant $(\mathbf{p}<0.05)$, \#\#\# very high significant $(P<0.001)$ vs. oxaliplatin group.

3.3. Histopathological results:

3.3.1 Right sciatic nerve tissues:

- Gross appearance:

The right sciatic nerve from each rat was pale yellow in color, cylindrical in shape, about 4-5 cm long, 20-30 mg weight, soft in consistency and had smooth surface. It was about $1 \mathrm{~mm}$ diameter by cross section. No gross abnormalities were detected in sciatic nerves dissected from rats of all groups.

- Light microscopic appearance: Control group and CONPs treated group showed, normal sciatic nerve tissues, formed of variable sized closely packed myelinated nerve fibers with endoneurium in between (figure 4).

Oxaliplatin treated group showed, mild to moderate demyelination and degeneration of myelin sheath which propagated in degree to be severe after 8 weeks with inflammation signs on top in some sections. Also, disturbed and remnant Schwann cells nuclei appeared in some sections (figure 4).

Oxaliplatin \& CONPs treated group showed, normal sciatic nerve structure after 4 and 8 weeks of the study (figure 5). 

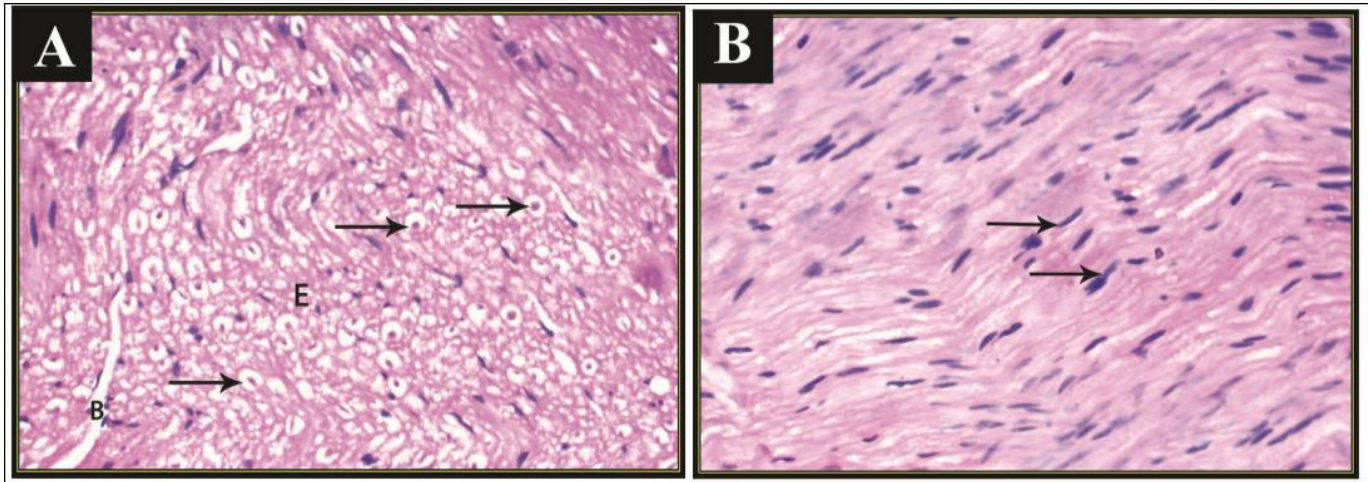

Figure (4): Representative photomicrographs of sciatic nerve sections from control group and CONPs treated group showing: (A) closely packed nerve fibers with endoneurium in between $(\mathrm{E})$ and an occasional endoneurial blood vessel (B). Each individual nerve fiber consists of central axon surrounded by myelin sheath $(\rightarrow)$. $($ B) the elongated wavy Schwann cell nuclei $(\rightarrow)($ H\&E x 400).
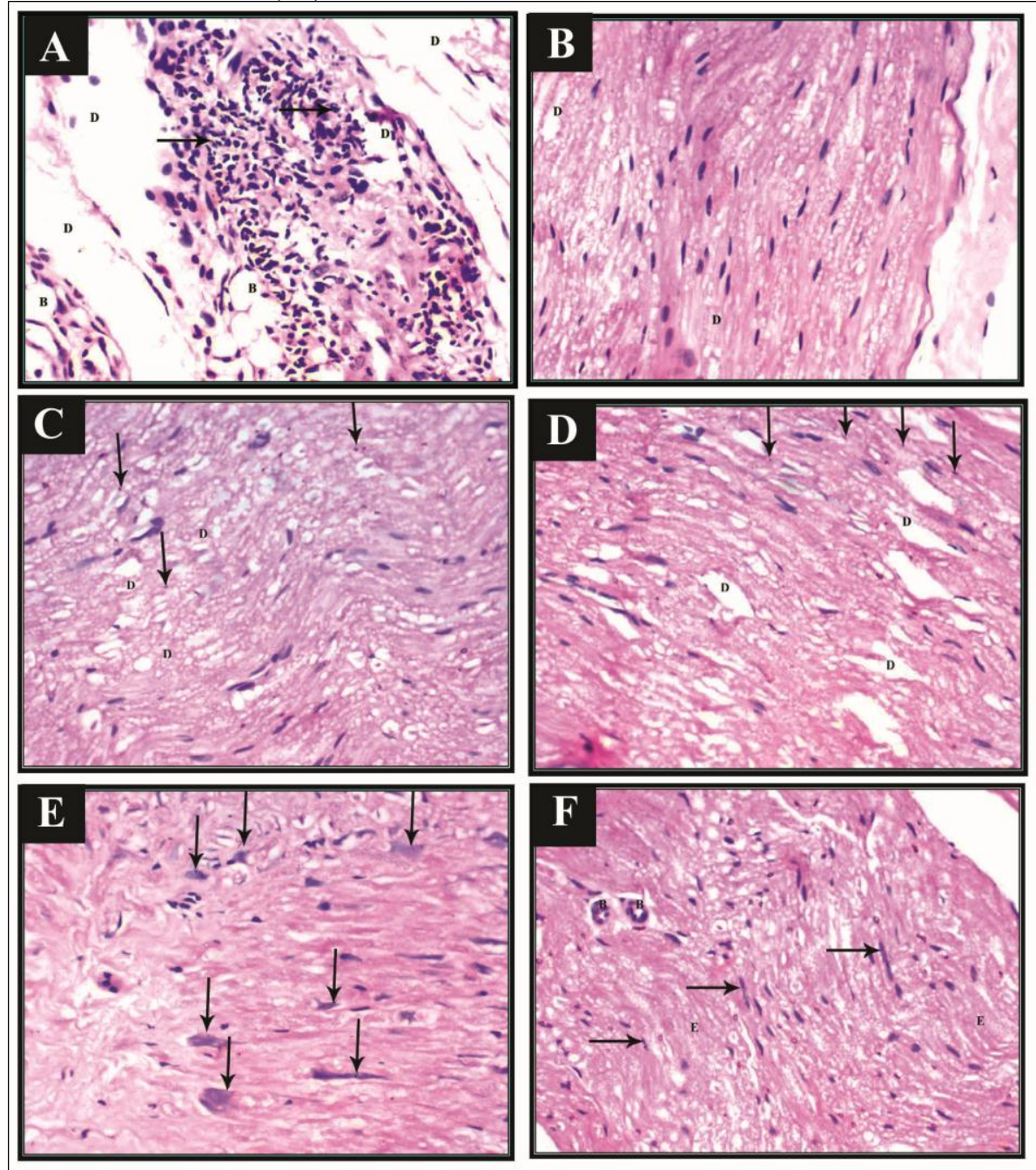

Figure (5): Representative photomicrographs of sciatic nerve sections from oxaliplatin (A, B, C, D\& E) and oxaliplatin\& CONPs trated group (F) showing: (A) Inflammation of sciatic nerve tissue $(\rightarrow$ ) on background of demyelination and degeneration (D) with increased number of blood vessels (B). (B) Mild, (C) Moderate , (D) Severe demyelination and degeneration of myelin sheath in some fibers with remnant of Schwann cells nuclei $(\downarrow)$.(E) disturbed Schwann cells nuclei $(\downarrow)$. $(\mathbf{F})$ normal myelinated nerve fibers, Schwann cell nuclei $(\rightarrow)$ with endoneurium (E) in between and occasional blood vessels (H\&E x 400). 


\subsection{2 lumbar spinal cord tissues:}

- Gross appearance:

The lumbar spinal cord from each rat was white in color, cylindrical in shape, about $2 \mathrm{~cm}$ long, 30-40 mg weight, soft in consistency and had smooth surface. It was about 3-5 mm diameter by cross section. No gross abnormalities were detected in lumbar spinal cord parts dissected from rats of all treated groups.

- Light microscopic appearance:

Control group and CONPs treated group showed normal morphological appearance of the lumbar spinal cord which was in the form of gray and white matter, central canal, neurons, each one with a cell body, nucleus, nucleolus, nissl granules and glial cells as shown in (figure $6)$.

Oxaliplatin treated group showed, aggregation of glial cells with apoptosis after 4 and 8 weeks of the study. Many degenerated neurons and smudged nuclei were seen also in some sections after 8 weeks of the study (figure 7).

Oxaliplatin \& CONPs treated group showed, normal morphological appearance with hydropic degeneration of some neurons in few sections after 4 and 8 weeks of the study (figure 7).
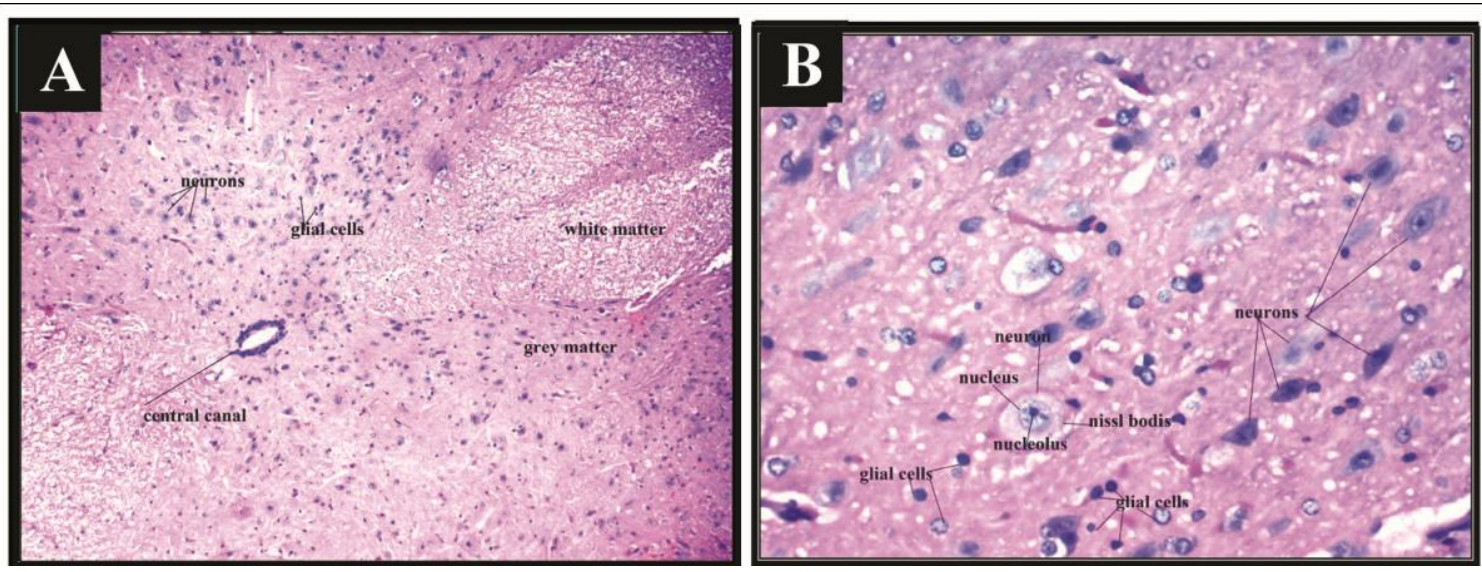

Figure (6): Representative photomicrographs of lumbar spinal cord sections from control group (A\&B) and CONPs treated group showing: normal morphological appearance (H\&E: A x 100; B x 400). 

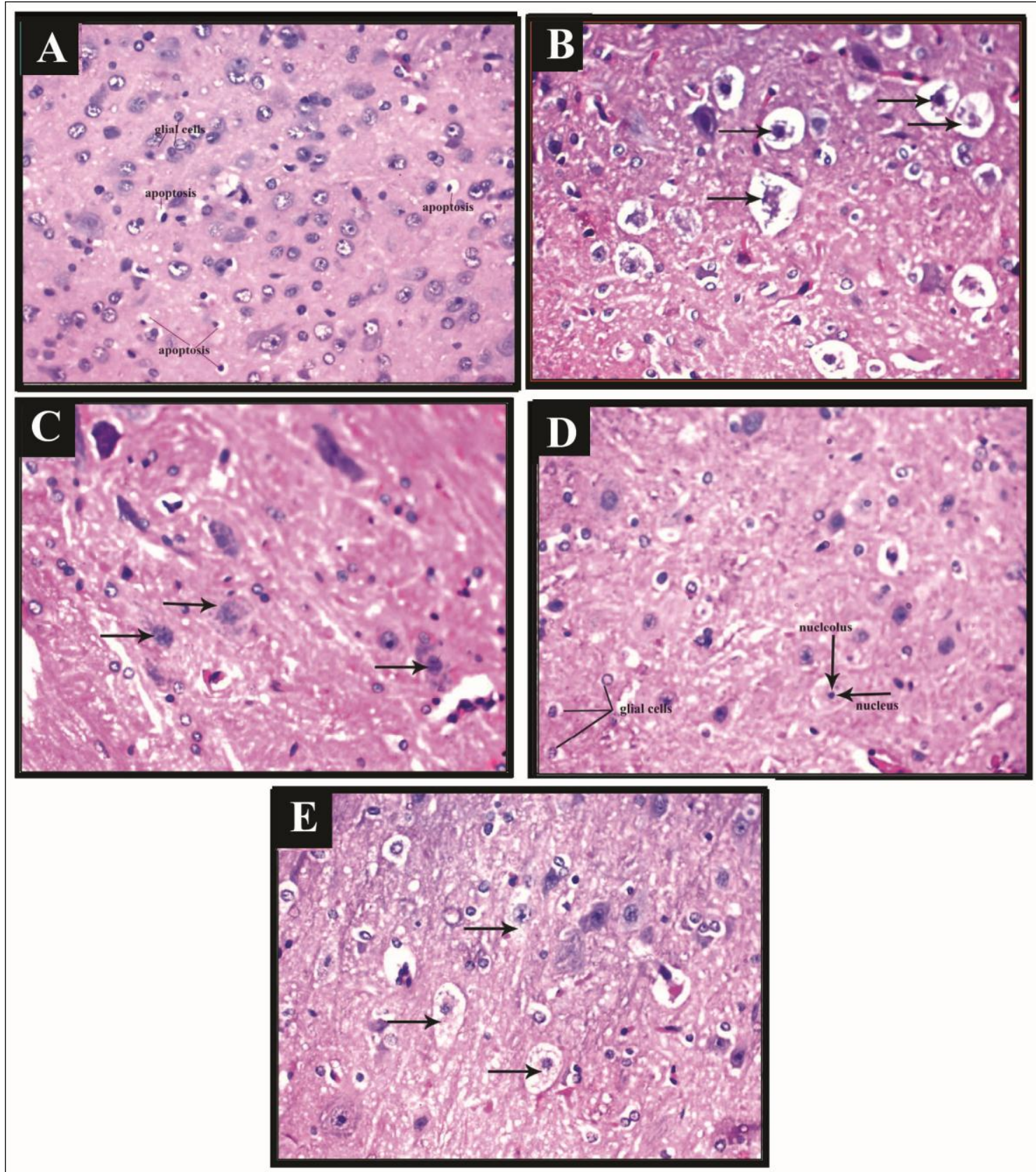

Figure (7): Representative photomicrographs of lumbar spinal cord sections from oxaliplatin (A, B \& C) and oxaliplatin\&CONPs trated group (D\&E) showing: (A) aggregation of glial cells with apoptosis. (B) degenerated neurons $(\rightarrow)$, (C) smudged nuclei $(\rightarrow)$ in neurons. (D) normal morphological appearance. (E) mild hydropic changes $(\rightarrow)(\mathbf{H} \& \mathbf{E} \mathbf{x} 400)$.

\section{DISCUSSION}

Oxaliplatin induced peripheral neuropathy occurs in about $60-80 \%$ of the patients treated with oxaliplatin and $15 \%$ of them develop severe forms (Sereno et al., 2017). Several pharmaceutical agents and nutraceuticals with antioxidant properties have been tested in vivo studies and in clinical trials to assess their effectiveness against peripheral neurotoxicity induced by oxaliplatin but no agent has proven to be effective (Cheng et al., 2017). CONPs are being proven promising for their neuroprotective and antioxidant properties (Heckman et al., 2013). Characterization of 
nanoparticles is essential for the studies as it has been shown that the size, shape, surface reactivity, solubility, and degree of aggregation are responsible for their differential response in biological system (Asati et al., 2010). The result of characterization of the current study were in the range of $<25 \mathrm{~nm}$ in size, cubes \& octahedrons in shape with Some agglomeration. This result was concomitant with Mittal and Pendy (2014) which used the same material.

The results of the present study showed that oxaliplatin decreased MPZ in sciatic nerve tissues after 4 and 8 weeks of the study. This finding was in agree with Kim et al. (2015) who found that MPZ immunoreactivity was decreased in the sciatic nerve sections of oxaliplatin treated group. Tsutsumi et al. (2014) reported that, oxaliplatin treatment reduced the levels of MPZ in sciatic nerves through decreasing the levels of cleaved neuregulin 1 (NRG1) type III protein in the sciatic nerves which is an important factor in the late phase of myelination. Moreover, gene expression of NRG1 and NRG1 mRNA levels were reduced by oxaliplatin in lumbar DRGs. CONPs administration with oxaliplatin showed significant increase in MPZ levels in the sciatic nerve tissues. These results are consistent with Hirst et al. (2011) who stated that, quenching ROS production capability of CONPs can decrease proteins destruction, lipid peroxidation, inflammation and subsequent tissue damage. Also the results of this study have been demonstrated that CONPs administration improve Schwann cells nuclei which become normal and thus have a good MPZ secretion.

$$
\text { Oxaliplatin injection caused a }
$$

significant increase in MDA level in sciatic nerves allover the period of the study. This result is concomitant with the result of Kim et al. (2015) who found that oxaliplatin injection induced increase in MDA levels in sciatic nerves of rats after 4 weeks of the study. This could be explained by Bertram and Hass, (2008) and Olmeda et al. (2008) who reported that, the activity of oxaliplatin drug is attributed to the formation of adducts with DNA that lead to intracellular generation of ROS following DNA and mitochondrial lesions. Areti et al. (2014) demonstrated that ROS can directly damage myelin sheath by oxidatively modifying fatty acid components of the phospholipid content of myelin leading to lipid peroxidation and formation of MDA.

Superoxide dismutase and GPx are important enzymes in the antioxidant system and their increase in Sciatic nerves by oxaliplatin after 4 weeks of the study suggests the enhancement of the antioxidant potential of the tissues to reduce oxidative stress (Hosseini et al., 2014). On the other hand, there was drastic fall in both enzymatic levels after 8 weeks of the study leading to conclusion that, the amount of oxidative stress was excessive to cause damage to these enzymes, resulting in the loss of antioxidant defenses (Areti et al., 2014). Oxaliplatin interacts with GSH and the depletion of GSH is one of the pathways through which platinum based cytotoxic drugs generate ROS in cells (Laurent et al., 2005). CONPs administration with oxaliplatin decreased the MDA levels in sciatic nerves of treated rats at 4 and 8 weeks of the study, which means that CONPs administration decreased the level of lipid peroxidation. Also, they decreased the levels of SOD and GPx enzymes activity induced by oxaliplatin after 4 weeks of the study which means that CONPs decrease oxidative stress in sciatic nerves. After 8 weeks of the study, CONPs improves significantly SOD enzyme activity but not significantly affect GPx enzyme activity.

Cerium oxide nanoparticles are potent antioxidants based on their ability to either donate or receive electrons as they alternate between the trivalent or tetravalent oxidation states. The catalytic properties of CONPs have been attributed to the presence of highly mobile lattice oxygen present at the surface, which facilitates the $\mathrm{Ce}^{4+}, \mathrm{Ce}^{3+}$ conversion and is correlated with decreased free radical levels (Heckman et al., 2013). Reduction of $\mathrm{Ce}^{4+}$ to 
$\mathrm{Ce}^{3+}$ causes oxygen vacancies and defects on the surface of the crystalline lattice structure of the nanoparticles, generating a cage for redox reactions to occur. They are able to scavenge superoxide, hydrogen peroxide and peroxynitrite. Accordingly, CONPs mimic the catalytic activities of antioxidant enzymes, such as SOD and catalase. Also, they are able to neutralize peroxynitrite (Pirmohamed et al., 2010; Dowding et al., 2012). Moreover, CONPs have several advantages over other pharmaceutical and nutraceutical antioxidants: first, CONPs act as catalysts to mimetic superoxide dismutase activity. Second, the antioxidant activity of these nanoparticles is mediated at oxygen vacancies on the surface. Therefore, one CONP offer many sites for catalysis because of its large surface/volume ratio, despite the fact that enzymes or pharmacological agents have only one active site per molecule. Finally, these nanoparticles remain resident and active in a living cell for an extended period of time (Hosseini et al., 2014).

The inability of CONPs to improve the reduction in GPx and GSH induced by oxaliplatin in sciatic nerves in the current work could be explained by McQuade et al. (2016) who reported that, oxaliplatin increased superoxide radicle particularly. Also, Rzigalinski et al. (2017) demonstrated that, if CONPs were in an area with high superoxide concentrations, more cerium would remain in the tetravalent state and $\mathrm{H}_{2} \mathrm{O}_{2}$ may accumulate. This accumulation will reduce GPx and GSH as, GPx is an essential antioxidant enzyme that converts $\mathrm{H}_{2} \mathrm{O}_{2}$ into water and $\mathrm{GSH}$ is an important cofactor of GPx to function in this reaction (Patil et al., 2007).

The histopathology results of sciatic nerve sections revealed that oxaliplatin treatment induced demyelination and degeneration of nerve fibers which was time dependent with inflammation. The Schwann cells were also affected as disturbed and their remnant nuclei were appeared in some sections. These results are in agree with previous studies. Al Moundhri et al. (2013) study showed that oxaliplatin induced focal areas of demyelination and degeneration of the sciatic nerve fibers of treated rats. Areas of infiltration with mononuclear inflammatory cells were also noticed. Kono et al. (2015) examined the sciatic nerves of oxaliplatin treated rats under electron microscope and showed that oxaliplatin induced damage of myelinated axons in the sciatic nerves in the form of accumulation of organelles, vacuolated swollen mitochondria and infiltration of macrophages. There was no notable morphological changes of non myelinated fibers of sciatic nerves. Inflammation of sciatic nerves in some sections in the current study could be explained by oxaliplatin generation of ROS. They activate various cellular signaling pathways that enhance the transcription of proinflammatory cytokines and chemokines such as tumor necrosis factor alpha (TNF- $\alpha$ ) and interleukins IL-1\& IL-6 (Oyenihi et al., 2015). As regard affection of Schwann cells by oxaliplatin administration, the findings of the present study are in agree with Imai et al. (2017) in vitro study who reported that exposure of primary cultured rat Schwann cells to oxaliplatin $(3 \mu \mathrm{M})$ for 48 hours reduced Myelin basic protein (MBP) expression and impaired the myelin forming Schwann cells with mitochondrial dysfunction. CONPs administration with oxaliplatin improved the myelination process as they reversed the histopathological and morphological abnormalities of sciatic nerves in treated rats. These findings are in agree with Kastrinaki et al. (2015) ex vivo study who showed that, CONPs translocated within the sciatic nerve of frogs by formation of narrow bands and this translocation depends on both axonal integrity and electrical activity. Nerve vitality curve comparison between control and exposed nerves showed that CONPs had no neurotoxic effect at the concentrations tested. Moreover Xu et al. (2016) reported that, CONPs administration decreased proinflammatory cytokines including IL- $1 \beta$, TNF$\alpha$ \& IL- 6 in serum and tissues of male mice exposed to oxidative stress and inflammation 
induced by air pollution model. CONPs decrease the inflammation of mice tissues by their antioxidant effect.

In the present study, aggregation of glial cells, many degenerated neurons, smudged nuclei and apoptosis were seen in the lumbar spinal cord sections from oxaliplatin treated group after 4 and 8 weeks of the study. The present results of lumbar spinal cord pathology are in agree with Sakurai et al. (2009) who reported that, oxaliplatin injection induced lesions of cell bodies, changes in the nucleus and nucleolus, atrophy in neurons of the DRG and cell death. Carozzi et al. (2015) demonstrated that platinum accumulation exerts cytotoxic effects in the DRG neurons by formation of inter and intra strand crosslinks in the DNA and accumulation of platinum mitochondrial DNA adducts lead to mitochondrial dysfunction with alteration in redox metabolism which induces DRG cells apoptosis. CONPs administration with oxaliplatin improves the morphological appearance of the lumbar spinal cord sections of treated rats as most sections showed normal morphological appearance. Few sections showed mild hydropic degeneration of neurons allover the period of the study. In an in vitro study by Dowding et al. (2014), CONPs are internalized by neurons after 3 hours and accumulate at plasma membrane and mitochondrial outer membrane. Furthermore, CONPs reduce levels of ROS in neurons exposed to oxidative stress. So, they reduce mitochondrial fragmentation and neuronal cell death. Najafi et al. (2017) also reported that, CONPs administration reversed the histopathology and morphological abnormalities of DRG neurons of diabetic rats leading to conclusion that CONPs reduced oxidative stress induced by degeneration of neurons and apoptosis as well as improve the antioxidant defense of tissues.

\section{ACKNOWLEGEMENTS}

A special thanks to my dear colleague Dr. Mona Atef lecturer of Forensic Medicine and Toxicology, Faculty of Medicine, Zagazig
University, for gifting me the animals for training on tissue dissection and nerve exploration before starting my actual work.

\section{REFERENCES}

Areti, A.; Yerra, V.G.; Naidu, V. et al. (2014): Oxidative stress and nerve damage: role in chemotherapy induced peripheral neuropathy. Redox Biology; 2: 289-295.

Argyriou, A.A. (2015): Updates on Oxaliplatin Induced Peripheral Neurotoxicity (OXAIPN). Toxics; 3: 187-197.

Arnold, M.; Sierra, M.S.; Laversanne, M. et al. (2017): Global patterns and trends in colorectal cancer incidence and mortality. Gut; 66(4): 683-691.

Asati, A.; Santra, S.; Kaittanis, C. et al. (2010): Surface charge dependent cell localization and cytotoxicity of cerium oxide nanoparticles. ACS Nano; 4(9): 5321-5331.

Bala, U.; Tan, K.; Ling, K. et al . (2104): Harvesting the maximum length of sciatic nerve from adult mice: a step-by-step approach. BioMed Central Research Notes; 7: 714.

Balayssac, D.; Ferrier, J.; Pereira, B. et al. (2015): Prevention of oxaliplatin induced peripheral neuropathy by a polyamine reduced diet-NEUROXAPOL: protocol of a prospective, randomised, controlled, singleblind and monocentric trial. The British Medical Journal; 5: e007479.

Bertram, C. and Hass, R. (2008): Cellular responses to reactive oxygen species-induced DNA damage and aging. Biological Chemistry; 389(3): 211-220.

Beutler, E.; Duron, O. and Kelly, M.B. (1963): Improved method for the determination of blood glutathione. Journal of Laboratory and Clinical Medicine; 61: 882-888.

Carozzi, V.A.; Canta, A. and Chiorazzi, A. (2015): Chemotherapy induced peripheral neuropathy: What do we know about mechanisms?. Neuroscience Letters; 596: 90107.

Cheng, X.; Huo, J.; Wang, D. et al. (2017): Herbal Medicine AC591 Prevents Oxaliplatin Induced Peripheral Neuropathy in Animal 
Model and Cancer Patients. Frontiers in Pharmacology; 8: 344.

Das, S.; Dowding, J.; Klump, K.; et al. (2013): Cerium oxide nanoparticles: applications and prospects in nanomedicine. Nanomedicine (London, England); 8 (9): 14831508.

Dowding, J.M.; Dosani, T.; Kumar, A. et al. (2012): Cerium oxide nanoparticles scavenge nitric oxide radical ( $\mathrm{NO}$ ). Chemical Communications (Cambridge, England); 48(40): 4896-4898.

Dowding, J.M.; Song, W.; Bossy, K. et al. (2014): Cerium oxide nanoparticles protect against $\mathrm{Ab}$ induced mitochondrial fragmentation and neuronal cell death. Cell Death and Differentiation; 21: 1622-1632.

Fardell, J.; Vardy, L. and Johnston, I. (2015): The long-term impact of oxaliplatin chemotherapy on rodent cognition and peripheral neuropathy. Behavioural Brain Research; 291: 80-88.

Heckman, K.L.; Decoteau, W.; Estevez, A. et al. (2013): Custom cerium oxide nanoparticles protect against a free radical mediated autoimmune degenerative disease in the brain. ACS Nano; 7(12): 10582-10596.

Hershman, D.L.; Lacchetti, C.; Loprinzi, C.L. et al. (2014): Prevention and management of chemotherapy induced peripheral neuropathy in survivors of adult cancers: American Society of Clinical Oncology clinical practice guideline. Journal of clinical Oncology; 32(18): 19411967.

Hirst, S.M.; Karakoti, A.; Singh, S. et al. (2011): Bio-distribution and in vivo antioxidant effects of cerium oxide nanoparticles in mice. Environmental Toxicology; 28(2): 107-118.

Horobin R.W. and Bancroft J.D. (1998): Hematoxylin and Eosin as an oversight stain .In: Troubleshooting Histology Stains. First edition Chapter 22 Churchil Livingston, Pearson Professional limited Press, New York, London, and Madrid Melbourne, Sanfrancisco and Tokyo. PP. 88-93.

Hosseini, A.; Baeeri, M.; Rahimifar, A. et al. (2014): Antiapoptotic effects of cerium oxide and yttrium oxide nanoparticles in isolated rat pancreatic islets. Human and Experimental Toxicology; 32: 544-553.

Imai, S.; Koyanagi1, M.; Azimi, Z. et al. (2017): Taxanes and platinum derivatives impair Schwann cells via distinct mechanisms. Scientific Reports; 7: 5947.

Institute of Laboratory Animal Resources (1996): Guide for the Care and Use of Laboratory Animals. ( $7^{\text {th }}$ ed.). National Academy Press; pp.125. ISBN 0-309-05377-3.

Kastrinaki, G.; Samsouris, C.; Kosmidis, E.K. et al. (2015): Assessing the axonal translocation of $\mathrm{CeO} 2$ and $\mathrm{SiO} 2$ nanoparticles in the sciatic nerve fibers of the frog: an ex vivo electrophysiological study. International Journal of Nanomedicine; 10: 7089-7096.

Kim, S.T.; Chung, Y.H.; Lee, H.S. et al. (2015): Protective effects of phosphatidylcholine on oxaliplatin-induced neuropathy in rats. Life Sciences; 130: 81-87.

Kono, T.; Suzuki, Y.; Mizuno, K. et al. (2015): Preventive effect of oral goshajinkigan on chronic oxaliplatin-induced hypoesthesia in rats. Scientific Reports; 5: 16078.

Laurent, A.; Nicco, C.; Chéreau, C. et al. (2005): Controlling tumor growth by modulating endogenous production of reactive oxygen species. Cancer Research; 65(3): 948956.

McQuade, R.M.; Carbone, S.E.; Stojanovska, V. et al. (2016): Role of oxidative stress in oxaliplatin-induced enteric neuropathy and colonic dysmotility in mice. British journal of Pharmacology; 173: 35023521.

Mittal, S. and Pandey, A.K. (2014): Cerium oxide nanoparticles induced toxicity in human lung cells: role of ROS mediated DNA damage and apoptosis. BioMed Research International; 2014: 891934.

Najafia, R.; Hosseinib, A.; Ghaznavic, H. et al. (2017): Neuroprotective effect of cerium oxide nanoparticles in a rat model of 
experimental diabetic neuropathy. Brain Research Bulletin; 131: 117-122.

Nigjeh, M.; Rahimifard, M.; Pourkhalili, N. et al. (2012): Multi-organ protective effects of cerium oxide nanoparticle/selenium in diabetic rats: evidence for more efficiency of nanocerium in comparison to metal form of cerium. Asian Journal of Animal and Veterinary advances; 7(7): 605-612.

Nishikimi, M.; Roa, N.A. and Yogi, K. (1972): The Occurrence of Supeoxide Anion in the Reaction of Reduced Phenazine Methosulfate and Molecular Oxygen. Biochemical and Biophysical Research Communications; 46: 849-854.

Ohkawa, H.; Ohishi, W. and Yagi, K. (1979): Assay for lipid peroxides in animal tissues by thiobarbituric acid reaction. Analytical Biochemistry; 95: 351-358.

Olmedo, D.G.; Tasat, D.R.; Evelson, P. et al. (2008): Biological response of tissues with macrophagic activity to titanium dioxide. Journal of Biomedical Materials Research. part A; 84(4):1087-1093.

Oyenihi, A.B.; Ayeleso, A.O.; Mukwevho, E. et al. (2015): Antioxidant strategies in the management of diabetic neuropathy. BioMed Research International; 2015: 515042.

Paglia, D.E. and Valentine, W.N. (1967): Studies on the quantitative and qualitative characterization of erythrocyte glutathione peroxidase. The Journal of Laboratory and Clinical Medicine; 70: 158-169.

Patil, S.B.; Kodliwadmath, M.V. and Kodliwadmath, S.M. (2007): Study of oxidative stress and enzymatic antioxidants in normal Pregnancy. Indian Journal of Clinical Biochemistry; 22: 135-137.
Pirmohamed, T.; Dowding, J.M.; Singh. S. et al. (2010): Nanoceria exhibit redox statedependent catalase mimetic activity. Chemical Communications (Camb); 46: 2736-2738.

Rzigalinski, B.A; Carfagna, C.S. and Ehrich, M. (2017): Cerium oxide nanoparticles in neuroprotection and considerations for efficacy and safety. Wiley Interdisciplinary Reviews. Nanomedicine and Nanobiotechnology; 9: 117.

Sakurai, M.; Egashira, N.; Kawashiri, T. et al. (2009): Oxaliplatin-induced neuropathy in the rat: involvement of oxalate in cold hyperalgesia but not mechanical allodynia. Pain; 147: 165-174.

Schmidt, S.D.; Mazzella, M.J.; Nixon, R.A. et al. (2012): $A \beta$ measurement by enzymelinked immunosorbent assay. Methods in Molecular Biology; 849:507-27.

Sereno, M.; Gerardo, G.; Rubio, J. et al. (2017): Genetic polymorphisms of SCN9A are associated with oxaliplatin-induced neuropathy. BioMed Central Cancer; 17: 63.

Tsutsumi, K.; Yamashita, Y.; Ushio, S. et al. (2014): Oxaliplatin induces hypomyelination and reduced neuregulin 1 expression in the rat sciatic nerve. Neuroscience Research; 80: 8690.

Wen, J.; Sun, D.; Tan, J. et al. (2015): A Consistent, Quantifiable, and Graded Rat Lumbosacral Spinal Cord Injury Model. Journal of Neurotrauma; 32(12): 875-892.

Xu, Z.; FangZhu, y.; FengChang, H. et al. (2016): Nanoceria restrains PM2.5-induced metabolic disorder and hypothalamus inflammation by inhibition of astrocytes activation related $\mathrm{NF}-\kappa \mathrm{B}$ pathway in $\mathrm{Nrf} 2$ deficient mice. Free Radical Biology and Medicine; 99: 259-272. 
دراسة التأثير الوقائى لجزيئات أكسيد السيريوم متناهية الدقة على التسمم العصبي الناتج عن الأوكزالبلاتين فى ذكور الجرذان

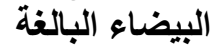

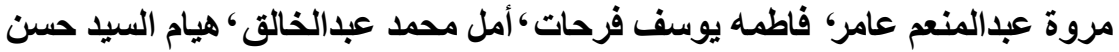

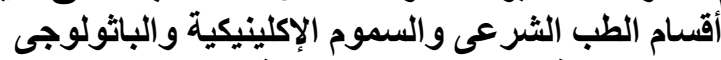

كلية الطب البشرى - جامعة الزقازيق الإنئي

الملخص العربى المبن

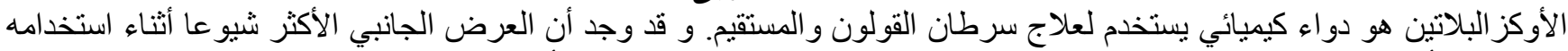

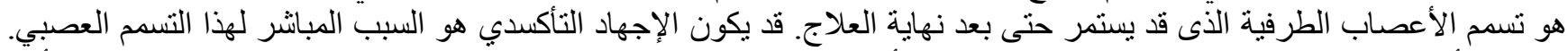

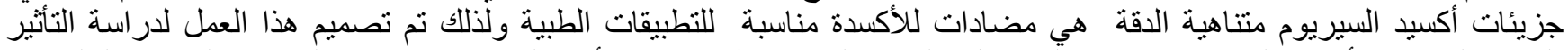

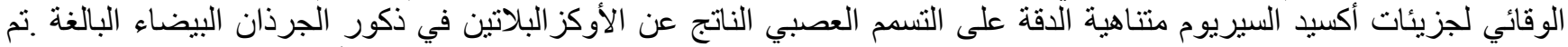

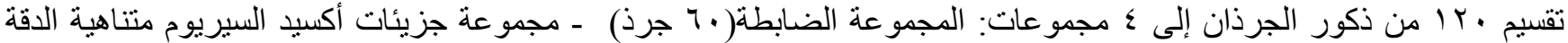

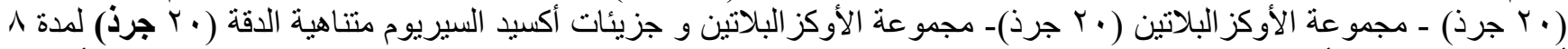

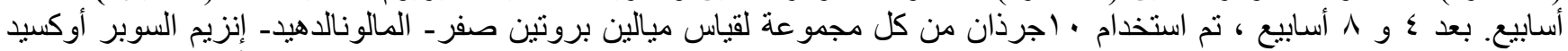

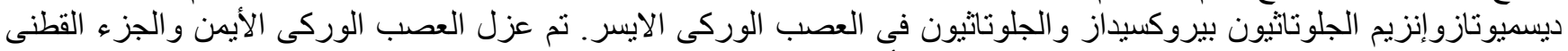

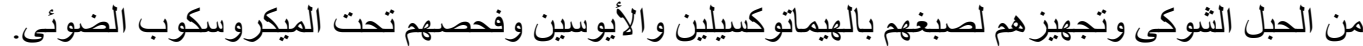

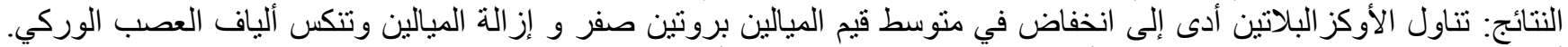

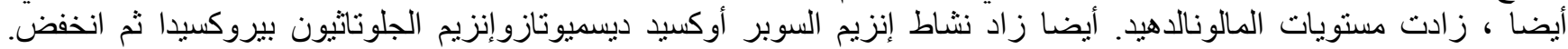

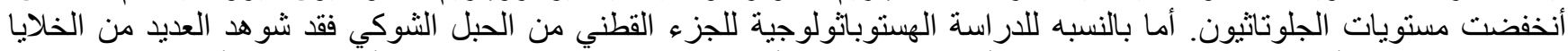

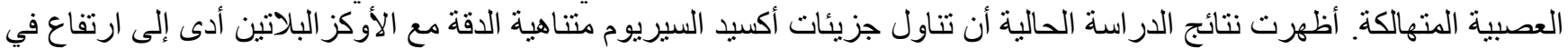

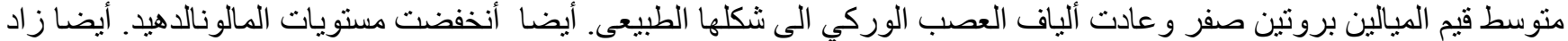

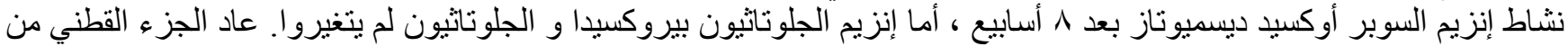

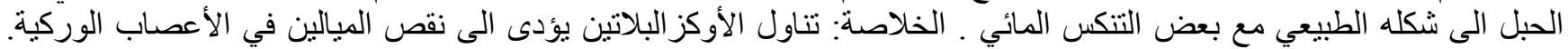
جزيئات أكسيد السيريوم متناهية الدقة لها تأثير الحماية العصبية. 